\title{
Modeling and Analysis of Normal Contact Stiffness of Machined Joint Surfaces
}

\author{
Hongping Yang \\ Tianshui Normal University, School of Engineering \\ ts1234@126.com
}

\begin{abstract}
This paper presents the mechanical joints normal contact stiffness model based on fractal geometry and contact mechanics theory. The joint normal dimensionless contact load and dimensionless contact stiffness relationship are analyzed in different fractal dimensions and materials. The result shows that relationship between joint normal contact load and contact stiffness plastic is the strongly nonlinear. At last, normal contact stiffness are compared and analyzed with the experimental values, as well as JZZ model used the present model. The comparison result indicates that the present model is consistent with experiment result.
\end{abstract}

Keywords:fractal geometry, mechanical theory, normal contact stiffness, modeling

\section{Introduction}

Machined joint exist extensively in machines and mechanical structures. The experimental results have shown that total deformation of the machine up to $90 \%$ is caused by joints [1-2]. There are several factors effect on joint characteristics such as surface processing methods, material and heat treatment method, surface roughness and surface integrity, joints type, size, shape, surface pressure and its distribution, joints media et. al.

The joints contact mechanism is very complex, and highly nonlinear [3-5]. Although many scholars tried to explain that some factors affect the joint surface stiffness from the microscopic mechanism qualitatively, but it is difficult to analyze and calculate the joints stiffness and damping for quantitative from the microscopic mechanism.

Greenwood and Williamson [6] proposed the GW joint contact theory based on fractal which has been applied widely by researchers. Onion and Archard [7] developed the OA model based on WA model. The calculation result is consistent with the GW model with separate relationship between the contact area, the contact load and the rough surface. Back [8] theoretically proved that the surface deformation and normal pressure relationship is the exponential function, and consistent with the experimental results.

The calculation results of statistical contact model which is associated with the instrumental resolution are of general uncertainty. The fractal contact models of selfaffine fractal geometry have unique deterministic analysis results. Fractal contact model includes MB model [9], improved MB model [10-11] and extended JS model [12].

Jiang [13] put forward the normal contact stiffness and tangential contact stiffness of machined joints using fractal contact theory. Shi [14] developed the joints normal contact stiffness theoretical calculation model using statistical contact theory. the contact parameters of a spherical slider and several different disk samples in contact 
with typical modern recording sliders were experimentally measured. Specifically, the contact stiffness and contact damping values were reported under different load, lubrication, and environmental conditions.

Zhao [15] presents a new elastic-plastic asperity microcontact model for contact between two nominally flat surfaces. The contacting asperity is transited from elastic deformation to fully plastic flow based on contact-mechanics theories in conjunction. The relations of the mean contact pressure and contact area of the asperity to its contact interference in the elastoplastic regime of deformation are respectively modeled. These asperity-scale equations are then used to develop the elastic-plastic contact model between two rough surfaces, allowing the mean surface separation and the real area of contact to be calculated as functions of the contact load and surface plasticity index. Results are presented for a wide range of contact load and plasticity index. It is important to accurately model the deformation in the elastoplastic transitional regime for the asperity contacts. However, the model is limited to the asperity elastic, elastoplastic, plastic contact deformation, contact area, contact load and other aspects of the research [16-17]. The relation between the surface normal contact stiffness and normal load was not discussed.

At present, there are two main aspects about the machined joints normal contact model for theoretical calculation. The first method is based on the theory of fractal geometry stiffness model, such as Majumder [9] made contact with fractal theory and fractal model for the contact. The second method is based on the theory of contact mechanics stiffness model.

This paper presents a new joint normal contact stiffness model combined fractal geometry theory and contact mechanical theory. The surface topography characteristic parameters are obtained by using fractal geometry theory. The surface normal contact stiffness model is obtained based on contact mechanics theory. The relationship is analyzed between normal contact stiffness load under different influent parameters. At last, the normal contact stiffness experimental results as a example, the theoretical model calculation results and the analysis are compared.

\section{Theoretical Background}

\subsection{Contact surface model}

The assumptions are the same as is made in GW [4] and CEB [18] model. The contact of two rough surfaces can be modeled by a flat, smooth surface in contact with a rough surface. Figure 1 shows an asperity contact with rigid smooth flat. Where, $y_{\mathrm{s}}$ is the distance between the mean of asperity heights and that of surface heights, $h$ is separation based on surface heights, $z$ and $d$ stand for the height of the asperity and the mean separation between the two surfaces, respectively. 


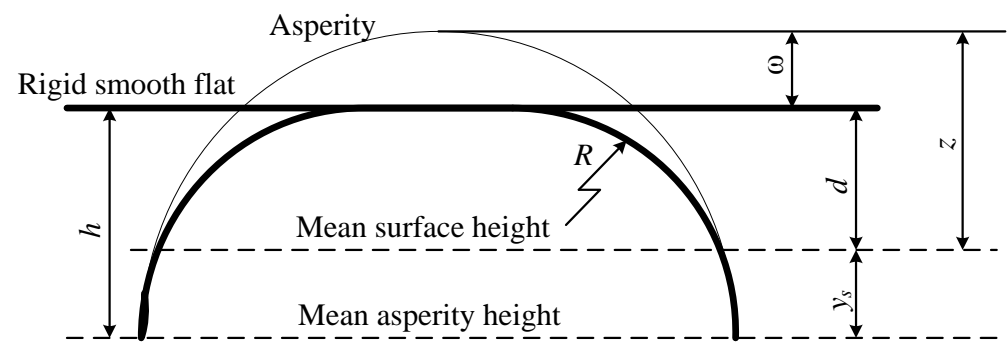

Figure 1. An Asperity Contact with Rigid Smooth Flat

The normal deformation is expressed as

$$
\omega=z-d
$$

\subsection{Characterization of surface asperities}

Fractal geometry can describe geometric features of various length scales, and thus provides a means of characterizing.

According to fractal theory, machining surface profile has non-stationary, selfsimilarity and multi-scale characterizations, its top surface asperities equivalent radius of curvature and height variance can be calculated, as in

$$
\begin{aligned}
& \sigma=G^{(D-1)} L^{(2-D)}(4-2 D)^{-1 / 2}(2 \ln \gamma)^{-1 / 2} \\
& R=2^{(2 D-4)} \pi^{-D / 2} G^{(1-D)}(\ln \gamma)^{-1 / 2}\left(a^{\prime}\right)^{D / 2} \\
& a^{\prime}=\left(\frac{2^{(9-2 D)} \pi^{(D-3)} G^{(2 D-2)} E^{2} \ln \gamma}{\lambda^{2} H^{2}}\right)^{1 /(D-1)}
\end{aligned}
$$

Where, $D$ denote the fractal dimension of a surface profile, $G$ denote the fractal roughness parameter, $\gamma$ is the scaling parameter for determining the spectral density and self-affine property, $L$ is the length of a fractal sample to be characterized. $E$ is a reduced elastic modulus, $E=\left[\left(1-v_{1}\right) / E_{1}+\left(1-v_{2}\right) / E_{2}\right]^{-1}$, where $v_{1}$ and $v_{2}$, and $E_{1}$ and $E_{2}$, are the Poisson ratios and elastic module of the two surfaces, respectively. $\sigma$ is surface asperity height variance, $R$ is the radius of curvature of contacting asperity on surface, $a^{\prime}$ is the intermediate parameter. $H$ is material hardness. $\lambda=0.454+0.41 v_{1}, v_{1}$ is the Poisson's ratio of the softer material.

\subsection{A single microcontact stiffness}

According to the Hertz contact theory, when asperity deformation $\omega$ is small, the elastic deformation is happened. With the asperity deformation increases, it will enter the plastic deformation stage, the elastic deformation of the elastic-plastic deformation changes the critical deformation $\omega_{\mathrm{e}}$, the expression is

$$
\omega_{\mathrm{e}}=\left(\frac{\pi C Y}{2 E}\right)^{2} R
$$


Where, $C=1.295 \exp (0.736 \mathrm{v}), Y$ denote material yield strength.

With $\omega$ increases, the asperity deformation will show three distinct stages: fully elastic, elastic-plastic and fully plastic. The following sections develop formulations for fully elastic, elastic-plastic, fully plastic deformation stage three contact load were $w_{\mathrm{e}}$, $w_{\mathrm{ep}}, w_{\mathrm{p}}$ as functions of $\omega$ during the three deformation stages. Studies shows[18] $\omega_{\mathrm{p}}=110 \omega_{\mathrm{e}}$.

$$
\begin{aligned}
& w_{\mathrm{e}}=\frac{4}{3} E R^{1 / 2} \omega^{3 / 2} \quad\left(\omega<\omega_{\mathrm{e}}\right) \\
& w_{\mathrm{ep}}=\pi H R \omega\left[1-1(1-\lambda) \frac{\ln \omega_{\mathrm{p}}-\ln \omega}{\ln \omega_{\mathrm{p}}-\ln \omega_{\mathrm{e}}}\right]\left[1-2\left(\frac{\omega-\omega_{\mathrm{e}}}{\omega_{\mathrm{p}}-\omega_{\mathrm{e}}}\right)^{3}+3\left(\frac{\omega-\omega_{\mathrm{e}}}{\omega_{\mathrm{p}}-\omega_{\mathrm{e}}}\right)^{2}\right] \quad\left(\omega_{\mathrm{e}} \leq \omega \leq \omega_{\mathrm{p}}\right) \\
& w_{\mathrm{p}}=2 \pi R H \omega \quad\left(\omega>\omega_{\mathrm{p}}\right)
\end{aligned}
$$

The corresponding contact stiffness is (9), (10), (11), respectively.

$$
\begin{aligned}
k_{\mathrm{e}}= & 2 E R^{1 / 2} \omega^{1 / 2} \quad\left(\omega<\omega_{\mathrm{e}}\right) \\
k_{\mathrm{ep}}= & \pi R H \omega\left[1-(1-\lambda) \frac{\ln \omega_{\mathrm{p}}-\ln \omega}{\ln \omega_{\mathrm{p}}-\ln \omega_{\mathrm{e}}}\right] f_{2}(\omega)+\pi R H\left[(1-\lambda) \frac{1}{\ln \omega_{\mathrm{p}}-\ln \omega_{\mathrm{e}}}\right] f_{1}(\omega) \\
& +\pi R H\left[1-(1-\lambda) \frac{\ln \omega_{\mathrm{p}}-\ln \omega}{\ln \omega_{\mathrm{p}}-\ln \omega_{\mathrm{e}}}\right] f_{1}(\omega) \quad\left(\omega_{\mathrm{e}} \leq \omega \leq \omega_{\mathrm{p}}\right) \\
k_{\mathrm{p}}= & 2 \pi R H \quad\left(\omega>\omega_{\mathrm{p}}\right)
\end{aligned}
$$

Where $f_{1}(\omega)=1-2\left(\frac{\omega-\omega_{\mathrm{e}}}{\omega_{\mathrm{p}}-\omega_{\mathrm{e}}}\right)^{3}+3\left(\frac{\omega-\omega_{\mathrm{e}}}{\omega_{\mathrm{p}}-\omega_{\mathrm{e}}}\right)^{2}$

$$
f_{2}(\omega)=6 \frac{\omega-\omega_{\mathrm{e}}}{\omega_{\mathrm{p}}-\omega_{\mathrm{e}}}-6\left(\frac{\omega-\omega_{\mathrm{e}}}{\omega_{\mathrm{p}}-\omega_{\mathrm{e}}}\right)^{2}
$$

\subsection{Two rough surface contact stiffness}

According to the above three kinds of asperity deformation mechanism, the two rough surface of the asperity contact is modeled. If the nominal contact area $A$ has asperities number $N, \eta, \Phi(z)$ are asperity distribution density, height distribution probability density function, the two surface contact load and stiffness is $W_{\mathrm{n}}, K_{\mathrm{n}}$. A surface for a given distance $d$, the normal contact stiffness $W_{\mathrm{n}}, K_{\mathrm{n}}$ can be expressed, respectively.

$$
\begin{aligned}
W_{n}(d)= & N \int_{d}^{d+\omega_{\mathrm{e}}} w_{\mathrm{e}} \Phi(z) d z+N \int_{d+\omega_{\mathrm{e}}}^{d+\omega_{\mathrm{p}}} w_{\mathrm{ep}} \Phi(z) d z+N \int_{d+\omega_{\mathrm{p}}}^{\infty} w_{\mathrm{p}} \Phi(z) d z \\
= & \frac{4}{3} \eta A E R^{0.5} \int_{d}^{d+\omega_{\mathrm{e}}} \omega^{1.5} \Phi(z) d z+\eta A \pi R H \int_{d+\omega_{\mathrm{e}}}^{d+\omega_{\mathrm{p}}} f_{1}(\omega)\left[1-(1-\lambda) \frac{\ln \omega_{\mathrm{p}}-\ln \omega}{\ln \omega_{\mathrm{p}}-\ln \omega_{\mathrm{e}}}\right] \omega \Phi(z) d z \\
& +2 \pi \eta A H R \int_{d+\omega_{\mathrm{p}}}^{\infty} \omega \Phi(z) d z
\end{aligned}
$$




$$
\begin{aligned}
K_{\mathrm{n}}(d)= & 2 \eta A E R^{0.5} \int_{d}^{d+\omega_{\mathrm{e}}} \omega^{0.5} \Phi(z) \mathrm{d} z+\eta A \pi R H \int_{d+\omega_{\mathrm{e}}}^{d+\omega_{\mathrm{p}}}\left[1-(1-\lambda) \frac{\ln \omega_{\mathrm{p}}-\ln \omega}{\ln \omega_{\mathrm{p}}-\ln \omega_{\mathrm{e}}}\right] f_{2}(\omega) \omega \Phi(z) \mathrm{d} z \\
& +\eta A \pi R H \int_{d+\omega_{\mathrm{e}}}^{d+\omega_{\mathrm{p}}} f_{1}(\omega)\left[(1-\lambda) \frac{1}{\ln \omega_{\mathrm{p}}-\ln \omega_{\mathrm{e}}}\right] \Phi(z) \mathrm{d} z \\
& +\eta A \pi R H \int_{d+\omega_{\mathrm{e}}}^{d+\omega_{\mathrm{p}}}\left[1-(1-\lambda) \frac{\ln \omega_{\mathrm{p}}-\ln \omega}{\ln \omega_{\mathrm{p}}-\ln \omega_{\mathrm{e}}}\right] f_{1}(\omega) \Phi(z) \mathrm{d} z \\
& +2 \pi \eta A R H \int_{d+\omega_{\mathrm{p}}}^{\infty} \Phi(z) \mathrm{d} z
\end{aligned}
$$

To make the model calculation results have wide versatility, the dimensionless $W_{\mathrm{n}}{ }^{*}, K_{\mathrm{n}}{ }^{*}$ equations are given

$$
\begin{aligned}
W_{n}^{*}= & \frac{4}{3} \beta\left(\frac{\sigma}{R}\right)^{0.5} \int_{h^{*}-y_{\mathrm{s}}^{*}}^{h^{*}-y_{\mathrm{s}}^{*}+\omega_{\mathrm{e}}^{*}} \omega^{* 3 / 2} \Phi^{*}\left(z^{*}\right) d z^{*}+\frac{\pi H \beta}{E} \int_{h^{*}-y_{\mathrm{s}}^{*}+\omega_{\mathrm{e}}^{*}}^{h^{*}-y_{\mathrm{s}}^{*}+\omega_{\mathrm{p}}^{*}} \omega^{*} f_{1}^{*}\left(\omega^{*}\right) f_{3}^{*}\left(\omega^{*}\right) \Phi^{*}\left(z^{*}\right) d z^{*} \\
& +\frac{2 \pi H \beta}{E} \int_{h^{*}-y_{\mathrm{s}}^{*}+\omega_{\mathrm{p}}^{*}}^{\infty} \omega^{*} \Phi^{*}\left(z^{*}\right) d z^{*} \\
K_{n}^{*}= & \frac{2 \beta \sqrt{A}}{\sqrt{\sigma R}} \int_{h^{*}-y_{\mathrm{s}}^{*}}^{h^{*}-y_{\mathrm{s}}^{*}+\omega_{\mathrm{e}}^{*}} \omega^{* 1 / 2} \Phi^{*}\left(z^{*}\right) d z^{*}+\frac{\pi \beta H \sqrt{A}}{E} \int_{h^{*}-y_{\mathrm{s}}^{*}+\omega_{\mathrm{e}}^{*}}^{h^{*}-y_{\mathrm{s}}^{*}+\omega_{\mathrm{p}}^{*}} f_{2}^{*}\left(\omega^{*}\right) f_{3}^{*}\left(\omega^{*}\right) \omega^{*} \Phi^{*}\left(z^{*}\right) d z^{*} \\
& +\frac{\pi \beta H \sqrt{A}}{E \sigma} \int_{h^{*}-y_{\mathrm{s}}^{*}+\omega_{\mathrm{e}}^{*}}^{h^{*}+y_{1}^{*}+\omega_{\mathrm{p}}^{*}} f_{1}^{*}\left(\omega^{*}\right) f_{4}^{*}\left(\omega^{*}\right) \Phi^{*}\left(z^{*}\right) d z^{*}+\frac{\pi \beta H \sqrt{A}}{E \sigma} \int_{h^{*}-y_{\mathrm{s}}^{*}+\omega_{\mathrm{e}}^{*}}^{h^{*}-y_{\mathrm{s}}^{*}+\omega_{\mathrm{p}}^{*}} f_{1}^{*}\left(\omega^{*}\right) f_{3}^{*}\left(\omega^{*}\right) \Phi^{*}\left(z^{*}\right) d z^{*} \\
& +\frac{2 \pi \beta H \sqrt{A}}{E \sigma} \int_{h^{*}-y_{\mathrm{s}}^{*}+\omega_{\mathrm{p}}^{*}}^{\infty} \Phi^{*}\left(z^{*}\right) d z^{*}
\end{aligned}
$$

Where $f_{1}^{*}\left(\omega^{*}\right)=1-2\left(\frac{\omega^{*}-\omega_{\mathrm{e}}^{*}}{\omega_{\mathrm{p}}^{*}-\omega_{\mathrm{e}}^{*}}\right)^{3}+3\left(\frac{\omega^{*}-\omega_{\mathrm{e}}^{*}}{\omega_{\mathrm{p}}^{*}-\omega_{\mathrm{e}}^{*}}\right)^{2}$,

$f_{2}^{*}\left(\omega^{*}\right)=6\left(\frac{\omega^{*}-\omega_{\mathrm{e}}^{*}}{\omega_{\mathrm{p}}^{*}-\omega_{\mathrm{e}}^{*}}\right)-6\left(\frac{\omega^{*}-\omega_{\mathrm{e}}^{*}}{\omega_{\mathrm{p}}^{*}-\omega_{\mathrm{e}}^{*}}\right)^{2}$

$f_{3}^{*}\left(\omega^{*}\right)=\left[1-(1-\lambda) \frac{\ln \omega_{\mathrm{p}}^{*}-\ln \omega^{*}}{\ln \omega_{\mathrm{p}}^{*}-\ln \omega_{\mathrm{e}}^{*}}\right]$

$f_{4}^{*}\left(\omega^{*}\right)=\left[(1-\lambda) \frac{1}{\ln \omega_{\mathrm{p}}^{*}-\ln \omega_{\mathrm{e}}^{*}}\right]$

$\omega^{*}=z^{*}-h^{*}+y_{\mathrm{s}}^{*}$

$y_{\mathrm{s}}^{*}=0.045944 / \beta$

$\beta=\eta \sigma R$

$\Phi^{*}\left(z^{*}\right)=1 / \sqrt{2 \pi}(\sigma / R) \exp \left[-0.5\left(\sigma / \sigma_{\mathrm{s}}\right)^{2} z^{* 2}\right]$ 


\section{Theoretical Results and Analysis}

In order to discuss the relationships characteristics of two rough surfaces contact stiffness and the load and deformation under certain conditions, assume two property parameters of the rough contact surfaces are: elastic modulus $E_{1}=E_{2}=207 \mathrm{GPa}$, hardness of softer material $H=1.96 \mathrm{GPa}$, Poisson's ratio $v_{1}=v_{2}=0.29$, fractal roughness parameter $G=5 \times 10^{-8}$.

\subsection{Analysis of the normal load on normal stiffness in different fractal dimension}

Figure 2 is given to the relationship between the dimensionless load and dimensionless stiffness in different fractal dimensions. It can be seen from the figures that the normal contact force and contact stiffness exhibit non-linear characteristics and exponential distribution relationships. The contact stiffness increases with the load increasing. Because the load is larger, the asperity contacts fully plastic deformation, which is consistent to the created mathematical model. It also can be seen from the figure that surface contact stiffness is increased with the fractal dimension increasing. When the contact load is smaller, the contact load and contact stiffness approximate linear relationship. It is because when the load is small, the surface asperity contact with the small number of asperity contact deformation is elastic deformation, but with normal load increases, the elastic contact with the actual surface area increases. When the fractal dimension is large, the surface roughness is small, the contact stiffness with the decrease of the surface roughness, which is also consistent with previous experimental results.

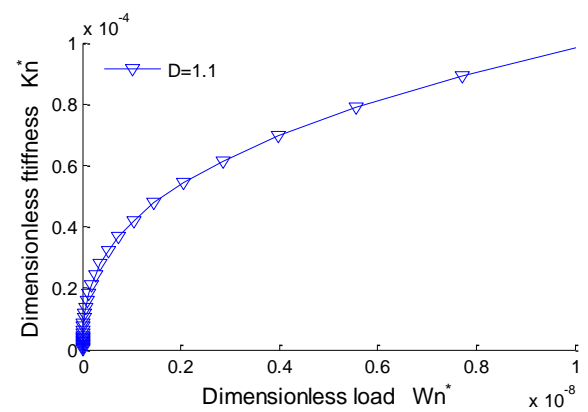

(a) $D=1.1$

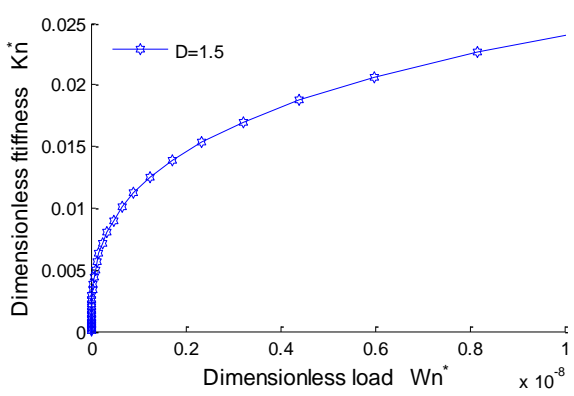

(b) $D=1.5$

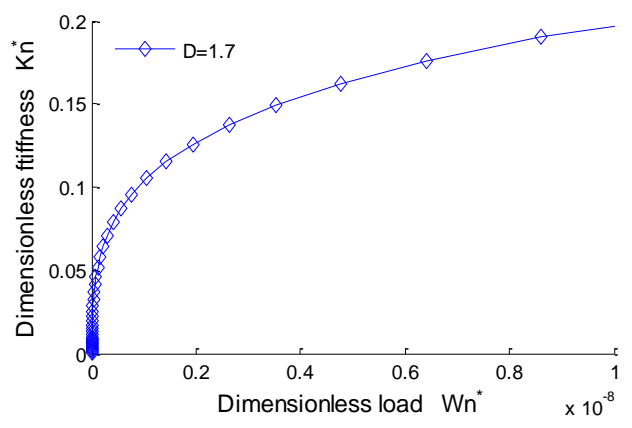




\begin{abstract}
(c) $D=1.7$
\end{abstract}

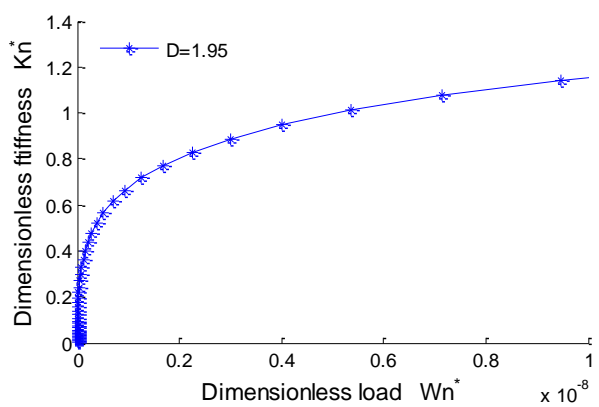

(d) $D=1.95$

Figure 2. The relationship between the normal load and stiffness in different fractal dimension

\title{
3.2. Analysis of the normal load on normal stiffness in different materials
}

The material elastic modulus is one of the important mechanical properties. In order to discuss the surface properties of materials effecting on joints normal stiffness, the following are analyzed in different elastic modulus of the material. The initial model parameters were as follows: the surface morphology of the fractal dimension $D=1.4$, scale factor $G=1 \times 10^{9}$.

Figure 3 presents the dimensionless normal load and normal contact stiffness curves in different surface material. From the figures, it can be seen that dimensionless normal stiffness increasing with dimensionless load increases, and the curves obey the nonlinear relationship. Comparing the figures of figure 3 (a) and (d), the dimensionless normal stiffness is higher in the same dimensionless load which the material elastic modulus is $E=7.2 \times 10^{10} \mathrm{~Pa}$ and $E=1.2 \times 10^{11} \mathrm{~Pa}$, respectively. The deformation stages of the fully elastic, elastic-plastic, fully plastic three performed prominent. It also can be seen that the normal stiffness and load is in linear relationship in the fully plastic deformation stage. It is because the larger of the elastic modulus, the greater of the contact stiffness, and the smaller of deformation.
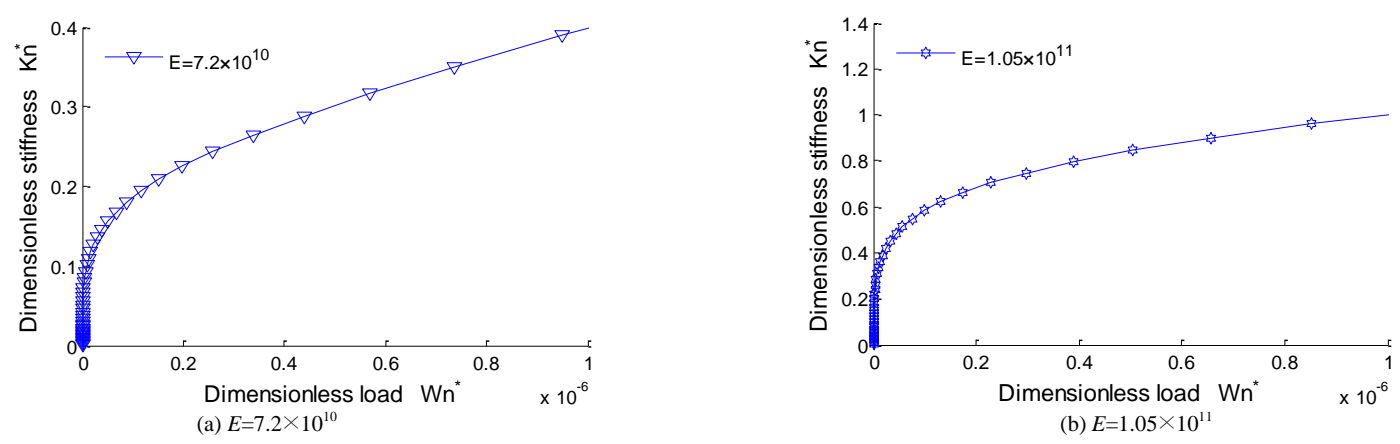

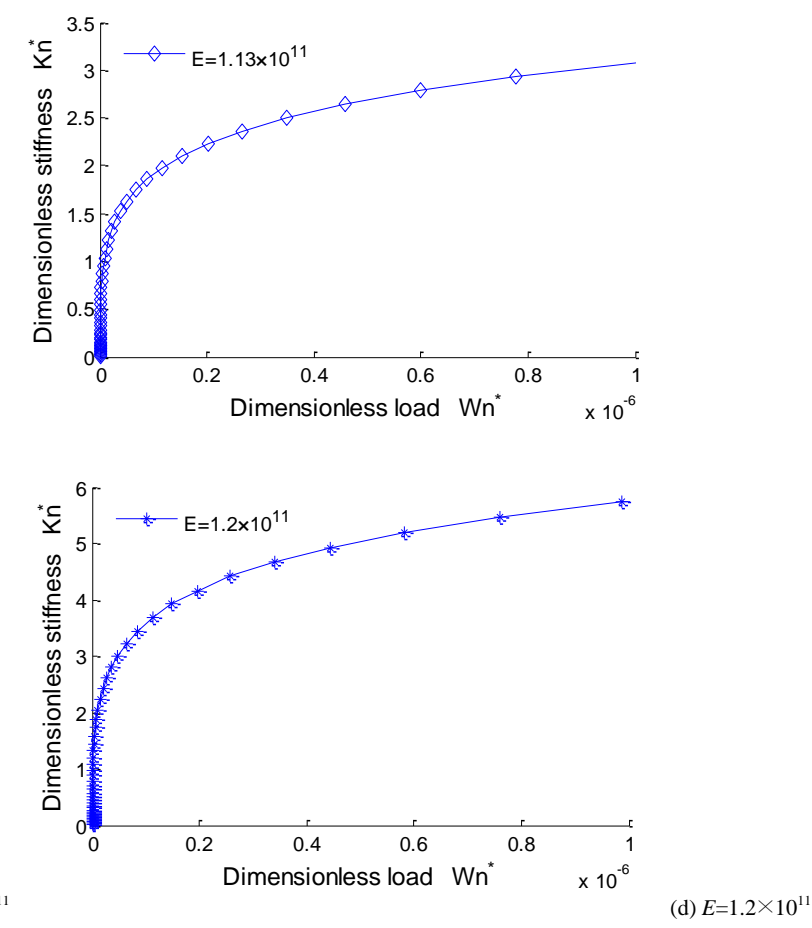

(c) $E=1.13 \times 10^{11}$

Figure 3. The Relationship between the Normal Load and Stiffness in Different Materials

\section{Experiment}

Although contact stiffness can be predicted from contact models as discussed in the previous section, there are numerous complexities involved, and it is necessary to experimentally measure for model validation.

\subsection{Testing}

A novel technique for measuring contact stiffness was used, as shown in Figure 4. The investigated interface is formed by the contact surfaces of area $2800 \mathrm{~mm}^{2}$ between the upper and the lower specimen, which is produced by milling. The kinds of the joint material are medium carbon steel. A dry joint surface is considered. The experimental parameters are surface fractal dimension $D=1.2526$, scale factor $G=1.403 \times 10^{-14}$, material elastic modulus is $E=2.1 \times 10^{11} \mathrm{~Pa}$. 


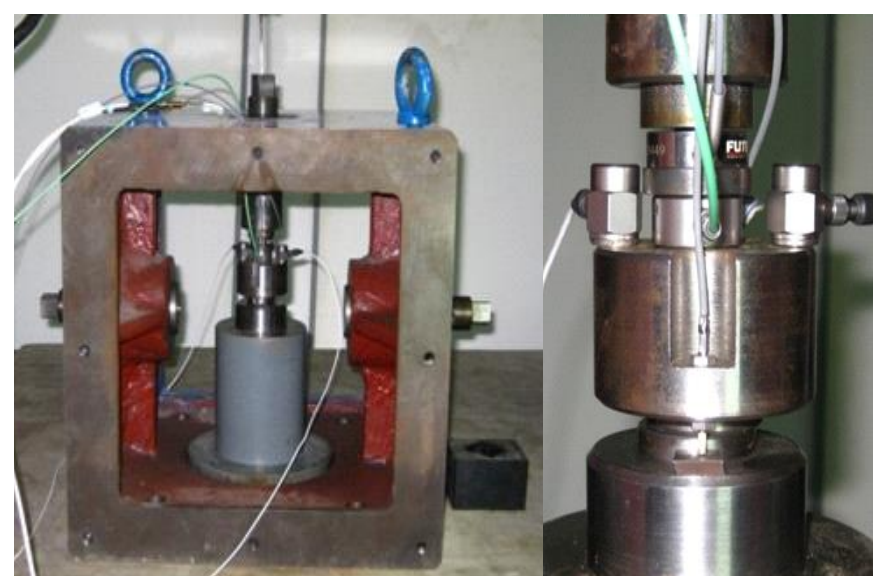

Figure 4. Contact Stiffness and Contact Damping Microtester

\subsection{Results and analysis}

The relationship between contact stiffness and contact load is nonlinear, as shown in Figure 5. Comparing with the experimental data and present model, the two curses are trended consistency. When normal contact loads less than $2.5 \mathrm{MPa}$, the experimental value larger than present model value. The two curses are close. With the normal load increase, that is normal contact load more than $2.5 \mathrm{MPa}$, the error value of experimental value and present model value getting bigger. Therefore, the following conclusions can be drawn that the model method of the contact stiffness calculation for the low surface load is more accurate. When the normal surface load is higher, the calculated value and the true value are larger. For another perspective, when the normal surface load is larger, the joint surface deformation has occurred, the asperity deformation has entered to fully plastic deformation stage, and however, the load part has failed.

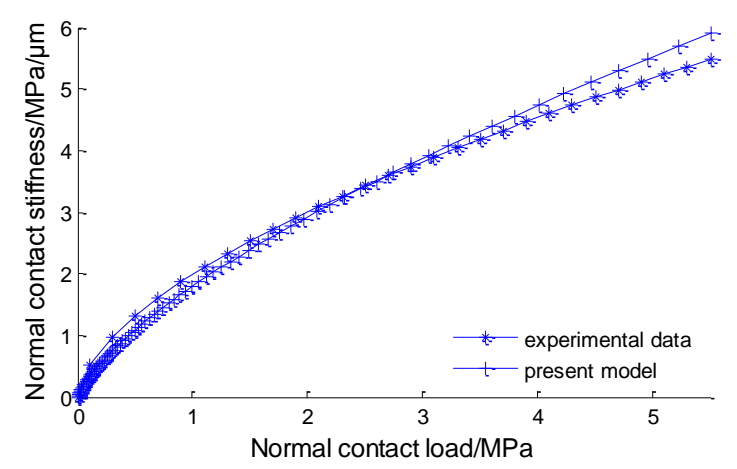

Figure 5. The Contact Stiffness Applied on Milling Joint

Jiang [13] established joint normal contact stiffness model based on fractal theory (JZZ model) and validated with experiments. The kinds of the joint material are cast iron. The contact surface was produced by grinding. Its parameters are as follows that elastic modulus $E=1 \times 10^{11} \mathrm{~Pa}$, the fractal dimension $D=1.2526$ and height scale parameter $G=1.403 \times 10^{-14}$, respectively.

To further verify the correctness of the present model, the values are compared for the JZZ model, experimental data, and present model in Figure 6. As can be seen from 
Figure 5, with the normal contact pressure increases, the model is consistent with the trend curve and the experimental curve. The present model calculated contact stiffness is close to the experimental values. However, the error value compared JZZ model with the experimental is growing. When normal load is less than $1.2 \mathrm{MPa}$, the present calculated value is greater than the experimental values. It can be concluded that the present model is a good method to calculate the joint normal contact stiffness.

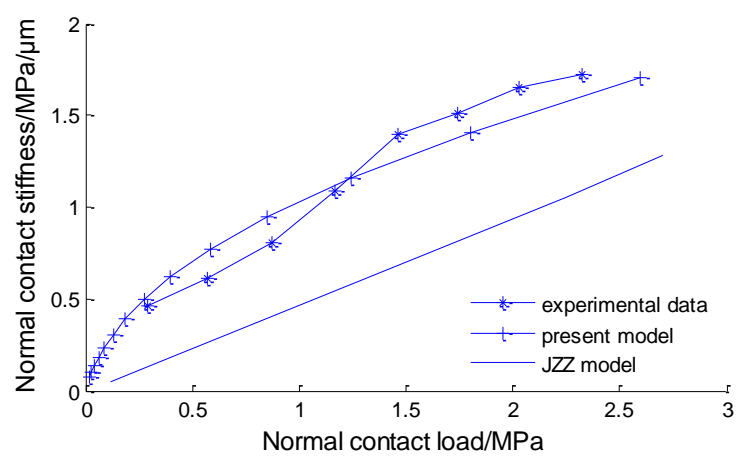

\section{Figure 6. The Contact Stiffness Applied on Milling Joint}

\section{Conclusion}

The results show that the joint normal contact stiffness is monotonically increases with normal contact load increases. The relationship of joint normal contact stiffness and normal contact load is nonlinear. The asperity deformation stages change from the fully elastic deformation, elastic-plastic to fully plastic. Compared with the experimental values, they are indicated that the present model for calculated the joint normal contact stiffness is rationality and effectiveness.

\section{Acknowledgements}

In this paper, the research was sponsored by the National Natural Science Foundation of China, the National Basic Research Program of China and the Tianshui Normal University Young Teachers Research Projects of China.

\section{References}

[1] R. H. Thornely and M. Khoyi, "The significance of joints and their orientation upon the overall deformation of some machine tool structure elements", M.T.D.R., (1970).

[2] R. L. Jackson and I. Green, "A finite element study of elasto-plastic hemispherical contact", ASME J. Tribol, vol. 127 , no. 2 , (2005), pp. 343-354.

[3] E. Ciulli, L. A. Ferreira and G. Pugliese, "Rough contacts between actual engineering surfaces Part II", Contact mechanics, Wear, vol. 264 (2008), pp. 1116-1128

[4] J. A. Greenwood and J. P. B. Williamson, "Contact of nominally flat surfaces", Proceedings of the Royal Society, vol. A952, (1966), pp. 300-319.

[5] R. Buczkowski and M. Kleiber, "Elasto-plastic statistical model of strongly anisotropic rough surfaces for finite element 3D-contact analysis", Comput. Methods Appl. Mech. Engrg, vol. 195, (2006), pp. 5141-5161.

[6] A. Majumdar and C. L. Tien, "Fractal characterization and simulation of rough surfaces", Wear, vol. 136, (1990), pp. 313-327.

[7] A. Majumdar and B. Bhushan, "Role of fractal geometry in roughness characterization and contact mechanics of surfaces", Tran of ASME, vol. 172, (1990), pp. 205-206.

[8] N. Baek, M. Burdekin and A. Cowley, "Review of the research on fixed sliding joint", Proe.13 ${ }^{\text {th }}$ Intern. Confer. M.T.D.R, vol. 85, no. 7, (1973), pp. 87-97. 
[9] A. Mmumdar and B. Bushan, "Fractal model of elastic-plastic contact between rough surfaces", ASME, Journal of Tribology, vol. 113, no. 1, (1991), pp. 1-11.

[10] S. Wang and K. Komvopoulos, "A fractal theory of the interfacial temperature distribution in the slow sliding regime: part I-elastic contact and heat transfer analysis", ASME Journal of Tribology, vol. 116, (1994), pp. 812-823.

[11] J. L. Liou and J. F. Lin, "A modified fractal microcontact model developed for asperity heights with variable morphology parameters", Wear, vol. 268, (2010), pp. 133-144.

[12] A. Goedecke, R. L. Jackson and R. Mock, "A fractal expansion of a three dimensional elastic-plastic multiscale rough surface contact model”, Tribology International, doi:10.1016/ j.triboint, vol. 2, no. 4, (2012).

[13] S. Y. Jiang, Y. J. Zheng and H. Zhu, "A contact stiffness model of machined plane joint based on fractal theory", ASME Journal of Tribology, vol. 132, (2010), pp. 1-7.

[14] X. Shi and A. A. Polycarpou, "Investigation of contact stiffness and contact damping for magnetic storage head-disk interfaces", ASME, Journal of Tribology, vol. 130, no. 021901, (2008), pp. 1-9.

[15] Y. W. Zhao and L. Chang, "A model of asperity interactions in elastic-plastic contact of rough surfaces", ASME, Journal of Tribology, vol. 123, (2001), pp. 857-864.

[16] L. Robert and L. Jeffrey, "A multi-scale model for contact between rough surfaces", Wear, vol. 261, (2006), pp. 1337-1347.

[17] L. Robert and I. Jackson, "A statistical model of elasto-plastic asperity contact between rough Surfaces", Journal of Tribology International Transactions, vol. 46, no. 3, (2006), pp. 906-914.

[18] Y. W. Zhao, M. David and L. Cang, "An asperity microcontact model incorporating the transition from elastic deformation to fully plastic flow”, ASME, Journal of Tribology, vol. 122, (2000), pp. 86-93. 
International Journal of Control and Automation Vol.7, No.6 (2014) 